\title{
Temperature Influence on Dielectric Response of Rotating Machine Insulation and Its Correction
}

\author{
J. Cheng ${ }^{1}$, P. Werelius ${ }^{2}$, N. Taylor ${ }^{1}$ \\ ${ }^{I}$ (School of Electrical Engineering and Computer Science, \\ KTH - Royal Institute of Technology, Stockholm, Sweden) \\ ${ }^{2}$ (Megger Sweden, Stockholm, Sweden) \\ jialu@kth.se
}

\begin{abstract}
Insulation condition is an essential aspect for the operational reliability of high voltage rotating machines in power plants and industrial applications. Insulation resistance (IR) and line-frequency dissipation-factor / power-factor $(\tan \delta)$ measurement are often performed for the assessment of stator insulation condition. These measured values need to be normalized to a reference temperature (e.g. $40{ }^{\circ} \mathrm{C}$ ) for comparison and trending and this is traditionally achieved by multiplying the results with a certain factor. However, this correction could be subject to error for an individual device since the correction factors recommended by various standards are average values of a certain number of machines at different conditions. In addition to that, insulation condition also has some influence on the temperature dependent property.

With the introduction of Dielectric Frequency Response, DFR and Polarization/Depolarization Current, PDC as more advanced insulation diagnostic methods, with proper modelling, temperature correction can be done based on the insulation condition of an individual device and thus accuracy is considerably improved. In this paper, the background of DFR and its superiority in temperature correction are introduced. After that, the numerical Fourier and Inverse Fourier Transformation algorithm is applied to correct the time domain measurement (IR and PDC).
\end{abstract}

\section{Introduction}

High voltage motors and generators are key components in power generation stations as well as other applications such as oil \& gas industry, steel company, pulp and paper industry. Any unexpected failure of those high voltage rotating machines may cause extensive damage to other apparatus, long time interruption of power supply and significant financial losses.

Effective maintenance strategy can reduce the risk of unexpected failure. According to a CIGRE survey [1], stator insulation is the component that has the highest failure rate for hydrogenerators. In general, the condition of stator insulation is poorly known, and appropriate electrical testing is required to detect potential deterioration and avoid catastrophic failure.

Insulation resistance, IR (including the polarization index) and dissipation factor / power factor / $\tan \delta$ test are the most widely used testing techniques all over the world. Temperature is an important factor that might change the results dramatically. Comparison of results over time needs to be performed without strong influence from extraneous parameters such as temperature. This can be done by controlling such parameters, or by compensating for their effect. Figure 1 is an example of trending the insulation resistance of a motor over time (two measurements per year performed in summer and winter respectively) with and without temperature compensation [2]. It indicates that without proper temperature correction, even a small fluctuation of temperature causes difficulty in detecting the insulation condition change. It happens frequently that people forget to record temperature during field insulation measurement. And fails to do so one is not able to be aware of the deterioration of stator insulation even if it is close to failure [3].

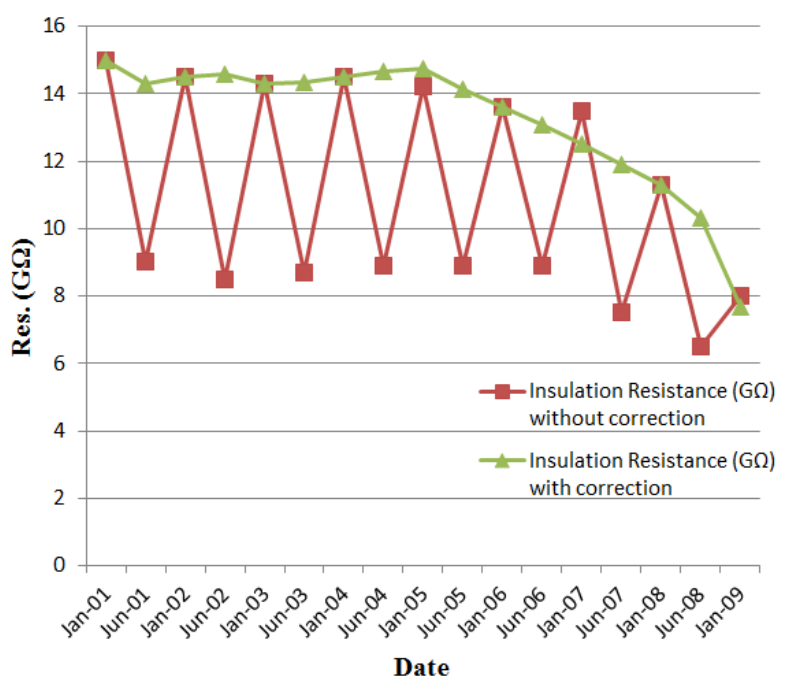

Figure 1. Insulation resistance graph with and without temperature correction

The commonly used temperature correction method has its limitations in aspect of accuracy. A more advanced correction algorithm for both IR and tan $\delta$ results is analyzed in this paper. However, due to the widely varying stator insulation materials and system designs, this paper puts more emphasis on the methodology of how to calculate the correction factor rather than giving correction factors for different insulation materials. 
By following the proposed procedures given by this paper, more reliable temperature correction is possible for various insulation materials.

\section{Typical temperature correction method}

It is common practice to do temperature correction by multiplying the testing result by a factor recommended by relevant standards. Table 1 is an example of commonly used temperature correction factors for insulation resistance measurement on rotating machines [3]. Such correction method can also be applied to $\tan \delta$ measurements. However, temperature correction factors in such table are usually average values of different materials and describe a material under "normal" conditions. In reality the condition of an insulation material is unknown and therefore the correction might be subject to error for an individual device [4].

Table 1 Approximate temperature correction factors for motor insulation resistance, from [3]

\begin{tabular}{|c|c|}
\hline Temperature $\left({ }^{\circ} \mathrm{C}\right)$ & Correction Factor $(\mathrm{Kt})$ \\
\hline 20 & 0.25 \\
\hline 30 & 0.50 \\
\hline 40 & 1.00 \\
\hline 50 & 2.00 \\
\hline 60 & 4.00 \\
\hline
\end{tabular}

The newer edition of the relevant standard C57.12.902010 [5] has the following note: "Experience has shown that the variation in power factor with temperature is substantial and erratic so that no single correction curve will fit all cases." The IR of a Class A rotating equipment at $50{ }^{\circ} \mathrm{C}$ differs by more than an order of magnitude as compared to that of $20{ }^{\circ} \mathrm{C}$. Therefore, if the given device does not conform to the "average" value then the applied correction factor of 10.45 might lead to a significant error in the estimation of the IR value at $20{ }^{\circ} \mathrm{C}$. Therefore, an individual approach for accurate temperature correction is of great benefit.

\section{Dielectric frequency response and its temperature characteristics}

\subsection{Dielectric frequency response}

With the development of modern electronics, newer technology enhances the performance of measurement circuit. It enables the tester to be able to measure the minuscule amounts of current that passes through the insulation system of a stator. Dielectric Frequency Response, DFR is a new testing technique of particular interest as it can yield greater insights into the insulation condition assessment.
The measurement principle and setup of DFR is very close to dissipation factor/power factor with the difference that insulation property is measured in much wider frequency range, typically from $1 \mathrm{mHz}$ to $1 \mathrm{kHz}$ rather than just at power frequency as shown in Figure 2. It is obvious that frequency sweep contains more information than just a single frequency measurement.

Another benefit of DFR is its easy-recognized temperature characteristics. It has been recorded by various literatures that the increase / decrease of temperature makes the curve shift towards higher/lower frequencies while the curve shape remains unchanged, as shown in Figure 2 [7].

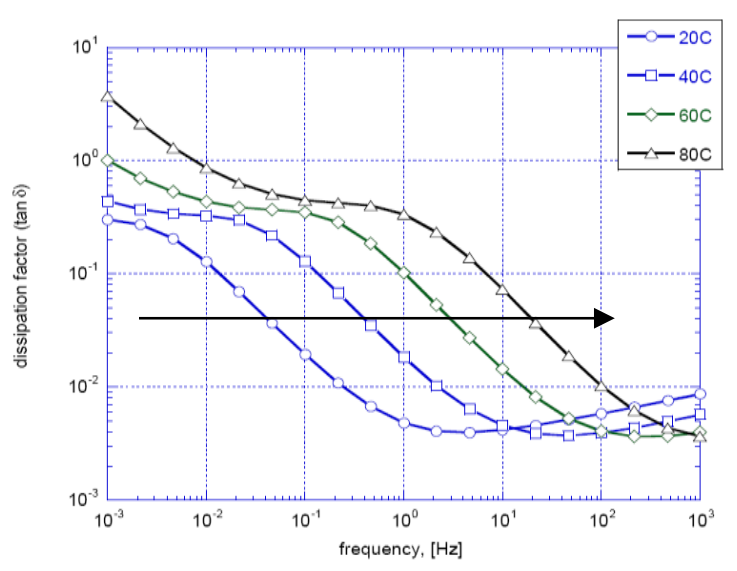

Figure 2. Typical curves of $\tan \delta$ vs. frequency. The arrow shows shifting of curves towards higher frequency at higher temperatures

\subsection{Individual temperature correction in frequency domain (AC)}

Instead of using average values, the individual temperature correction, ITC algorithm is built on the basis of actual temperature behavior of the insulation material and takes its condition into account [6]. Therefore it is able to provide temperature correction for an individual device in a more reliable way.

Taking the simplest dielectric property Debye relaxation as an example, its temperature dependent complex permittivity can be written as:

$$
\varepsilon=\varepsilon_{\infty}+\frac{\Delta \varepsilon}{1+j \omega \tau e^{-E_{a} /\left(k_{B} \cdot T\right)}}
$$

where $E a$ is the activation energy of the insulation material (J), $k_{B}$ is the Boltzmann constant $\left(1.38 \times 10^{-23} \mathrm{~J} / \mathrm{K}\right)$ and $T$ is the temperature $(\mathrm{K})$ of the object.

The activation energy can be determined by horizontally shifting complex relative permittivity curves obtained from various temperatures until a roughly continuous curve is 
constructed at a chosen reference temperature [8]. This curve is the so called "Master Curve".

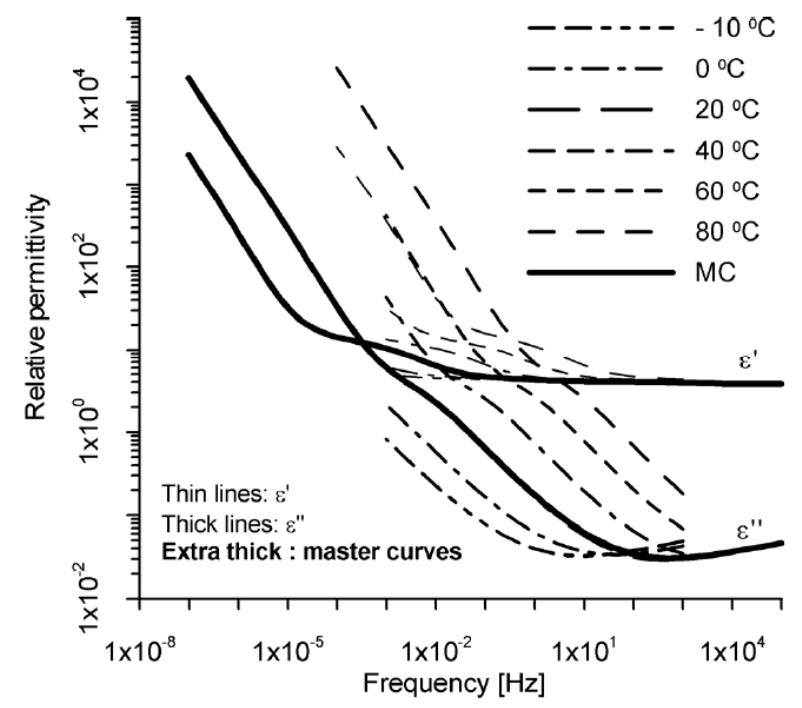

Figure 3. Formation of the Master Curve from a set of DFR measurements on one object at at different temperatures

After that, the shifted distance in logarithm scale is plotted against the multiplicative inverse of temperature. The slope of the straight line (absolute value) in Figure 4 is equal to the activation energy.

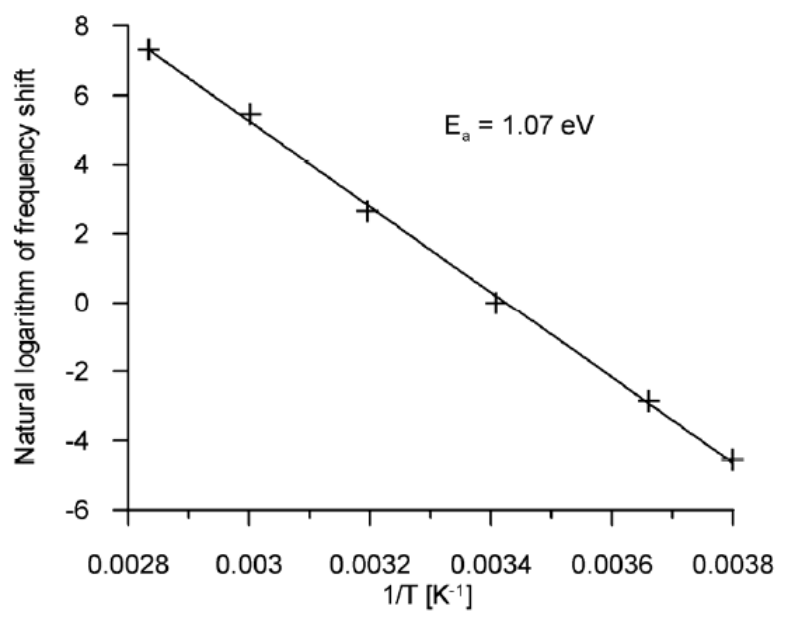

Figure 4. Plotting of shifted distance vs. reciprocal for temperature

Knowing the activation energy, the correction of measured DFR curve from temperature $T 1$ to temperature $T_{2}$ could be achieved by shifting the curve in logarithm scale by distance $\mathrm{L}$, which is equal to:

$$
L=\ln \left[-\frac{E_{a}}{k_{B}}\left(\frac{1}{T_{2}}-\frac{1}{T_{1}}\right)\right]
$$

\section{Polarization and depolarization current and its temperature characteristics}

\subsection{Individual temperature correction in time domain (DC)}

In time domain, insulation testing is performed by applying a DC voltage to the test object and recording the current with respect to time. The accurate correction of time domain data is difficult because that the magnitude of current/resistance curve also varies with temperature.

Fortunately, it is possible to transform the time domain data into frequency domain by Fourier Transformation. Suppose that the current vs. time curve has been obtained with object temperature $60{ }^{\circ} \mathrm{C}$, the correction to $40{ }^{\circ} \mathrm{C}$ can be achieved by following procedures:

- Convert the time domain data obtained at $60{ }^{\circ} \mathrm{C}\left(\mathrm{T}_{1}\right)$ into frequency domain $\left(\mathrm{F}_{1}\right)$ using the Fourier Transformation

- Apply individual temperature correction algorithm to the frequency domain data $\left(\mathrm{F}_{1}\right)$ so that frequency domain data at $40{ }^{\circ} \mathrm{C}\left(\mathrm{F}_{2}\right)$ is obtained

- Transform the frequency domain data $\left(\mathrm{F}_{2}\right)$ back into the time domain $\left(\mathrm{T}_{2}\right)$ using the inverse Fourier Transformation

\subsection{Fourier and inverse Fourier Transformation}

Based on [9] and neglecting some components that approaches zero when either time or frequency goes to zero and infinity, the time domain data and frequency domain data can be transformed into each other by using following equations:

$$
\begin{aligned}
& \mathrm{C}^{\prime \prime}(\omega)=\frac{1}{\mathrm{U}_{0}} \int_{0}^{\infty} \mathrm{i}_{\mathrm{pol}}(\mathrm{t}) \sin (\omega t) d t \\
& \mathrm{i}_{\mathrm{pol}}(\mathrm{t})=\frac{2}{\pi} \mathrm{U}_{0} \int_{0}^{\infty} \mathrm{C}^{\prime \prime}(\omega) \sin (\omega \mathrm{t}) \mathrm{d} \omega
\end{aligned}
$$

where $\omega$ is the angular frequency, $C^{\prime \prime}$ is the loss of dielectric material, $\mathrm{i}_{\text {pol }}(\mathrm{t})$ is the insulation leakage current during charging.

Since it is nearly impossible to obtain the analytical solution of the integration above, some approximation methods especially the Hamon approximation [10] are often used to simplify the transformations. Some error might be introduced and in addition to that, it often causes hesitation when giving a value to some unknown parameters. The numerical solution which can be expressed by equation (5) and (6) is a very good candidate for high quality transformation.

$$
C^{\prime \prime}(\omega)=\frac{1}{U_{0}} \sum_{n=0}^{\infty} i_{p o l}\left(t_{n}\right) \int_{t_{n}}^{t_{n}+\Delta t} \sin (\omega t) d t
$$




$$
\mathrm{i}_{\mathrm{pol}}(\mathrm{t})=\frac{2}{\pi} \mathrm{U}_{0} \sum_{\mathrm{n}=0}^{\infty} \mathrm{C}^{\prime \prime}\left(\omega_{n}\right) \int_{\omega_{n}}^{\omega_{n}+\Delta \omega} \sin (\omega \mathrm{t}) \mathrm{d} \omega
$$

The minimum time interval $(\Delta t)$ of two adjacent PDC measurement points is 1 second due to the instrument limitation and the first point is assumed to be captured at the first second since start of measurement. The missing data between time zero and the first second doesn't have much influence on the frequency range of interest. So expression (5) could be re-written to equation (7):

$$
C^{\prime \prime}(\omega)=\frac{1}{\mathrm{U}_{0}} \sum_{\mathrm{n}=0}^{\infty} \frac{\mathrm{i}_{\mathrm{pol}}\left(\mathrm{t}_{\mathrm{n}}\right)}{\omega}\left[\cos \left(\omega t_{n}\right)-\cos \left(\omega t_{n+1}\right)\right]
$$

where $t_{n}=n+1$. Selection of angular frequency $\omega$ is set according to the total measurement time $t_{\text {pol }}$ following equation (8) and (9):

$$
\begin{array}{cc}
\omega=n \pi / t_{\text {pol }} & \mathrm{n}=2,3,4 \ldots \\
\omega=\frac{2 n+1}{2} \pi / t_{\text {pol }} & \mathrm{n}=2,3,4 \ldots \ldots
\end{array}
$$

The purpose of such selection is to eliminate the fluctuation introduced by using a fixed current between two time points.

A $110 / 10 \mathrm{kV}$ transformer is measured in frequency domain (DFR) at $140 \mathrm{~V}$ RMS and time domain (PDC) at $100 \mathrm{~V}$ respectively. Then results are transformed into the other domain respectively and compared with the actual measured data. It can be observed from Figure 5 and Figure 6 that good agreement has been achieved for both transformations. To ensure accurate transformation, the time domain measurement should last for at least $1000 \mathrm{~s}$ and the frequency domain measurement should go down to $0.1 \mathrm{mHz}$. Although the transformation is only valid for a certain frequency and time range, this range is of particular interest for condition diagnosis.

\section{Non-linear system}

Linear insulation material exhibits identical dielectric property at different voltages. But almost all insulation materials or systems are more or less non-linear. For HV rotating machines coils, the stress grading material at the slot end makes the stator insulation system non-linear. The nonlinear property has been studied thoroughly and it causes difficulty in results interpretation and diagnosis [11].

Figure 7 is the DFR of an aged motor winding coil measured at different voltages. In lower voltage limit the non-linear property of the system is not obvious. With increased voltage levels, significant non-linear behavior is present.

Therefore, to reach the balance between measurement accuracy and maintaining linear property, test voltage between $1 \mathrm{kV}$ and $2 \mathrm{kV}$ is recommended for measurement that needs temperature correction afterwards. Fourier Transformation of the time domain result measured at $2 \mathrm{kV}$ DC is shown in Figure 8. The comparison of transformed data and actual measurement data suggests that accuracy is satisfactory.

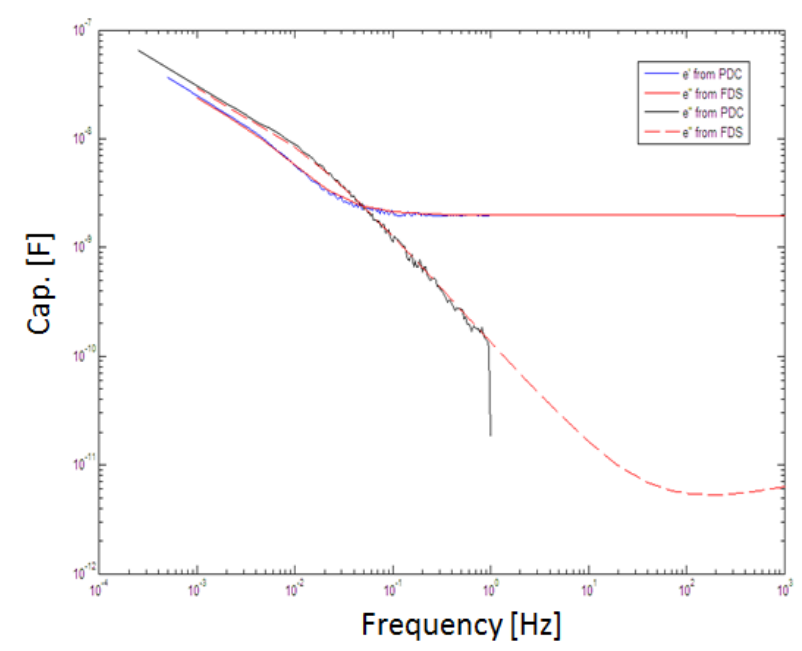

Figure 5. Transformation from time domain to frequency domain. The red curves are reference curves obtained from actual measurement. Blue and black curves are transformed curves from time domain data.

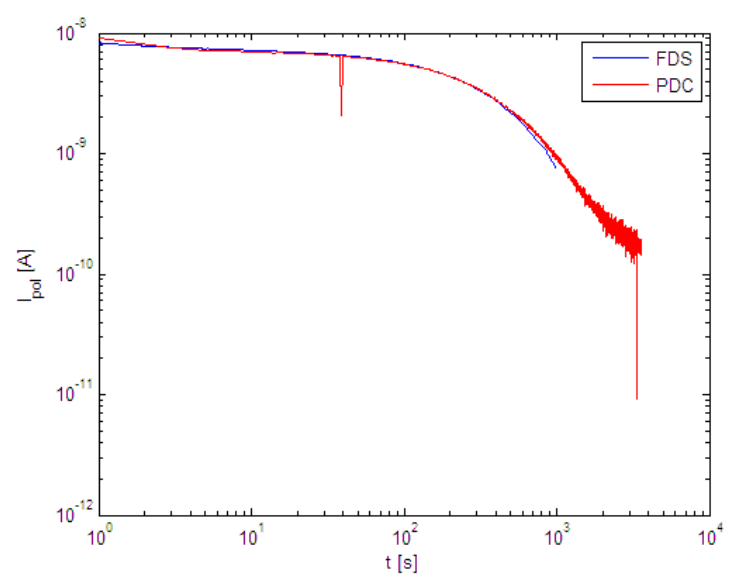

Figure 6. Transformation from frequency domain to time domain. The red curve is the reference curve obtained from actual measurement. Blue curve is the transformed curve from frequency domain data. 


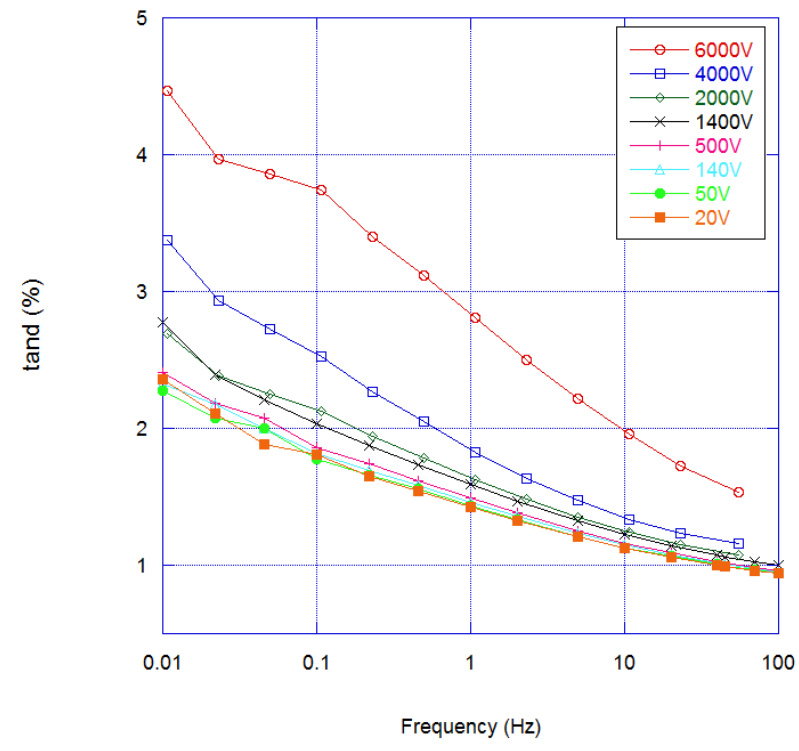

Figure 7. DFR of the aged motor coil at various AC voltages

\section{Conclusion}

Temperature correction can be achieved accurately in frequency domain by shifting the dielectric frequency response of an individual insulation material with certain distance. Good accuracy of numerical Fourier and inverse Fourier Transformation makes it possible to transform the time domain measurement into frequency domain and vice versa for the particular insulation material studied. Therefore, the time domain temperature correction can be achieved by transforming it into the frequency domain for ITC and then transforming back. In this way, the change of insulation condition over time can be identified more reliably and without confusion.

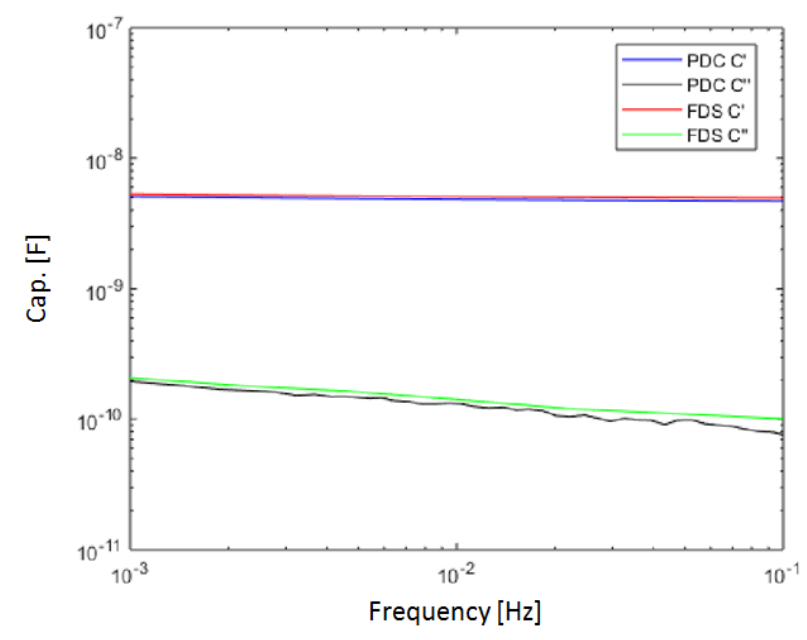

Figure 8. Fourier transformation of time domain measurement on the aged motor coil

\section{References}

[1] CIGRE Working Group A1.10, "Survey of Hydrogenerator Failures", 2009, pp. 5-9.

[2] Application Guide, A Guide to Diagnostic Insulation Testing Above $1 \mathrm{kV}$, Megger Ltd., Dover, 2017, pp. 25-26.

[3] IEEE Standard 43 - 2000, "IEEE Recommended Practice for Testing Insulation Resistance of Rotating Machinery", Electric Machinery Committee of IEEE Power Engineering Society, 2006.

[4] S. Zurek, A.E. Rasheed and M. Ohlen, "Individual Temperature Correction (ITC) for Insulation Resistance Measurements", 13th International Electrical Insulation Conference, 2017.

[5] R.K. Tyagi, S. Victor and N.S. Sodha, "Application of Temperature Correction Factors for Dissipation Factor Measurements for Power Transformers - A Case Study", Doble Client Conference, 2006.

[6] D. Robalino, P. Werelius, M. Ohlen and J. Cheng, "Dielectric Frequency Response Measurements and Dissipation Factor Temperature Dependence", The International Symposium on Electrical Insulation Conference, 2012.

[7] R. Neimanis, T. Saha, and R. Eriksson, "Determination of moisture content in mass impregnated cable insulation using low frequency dielectric spectroscopy", IEEE Power Engineering Society Summer Meeting, 2000.

[8] D. Linhjell, L. Lundgaard and U. Gäfvert, "Dielectric Response of Mineral Oil Impregnated Cellulose and the Impact of Aging", IEEE Transactions on Dielectrics and Electrical Insulation, Volume: 14 Issue:1, 2007.

[9] A. Helgesson, "Dielectric Properties of Machine Insulation studied with Dielectric Response", Licentiate thesis, KTH, Stockholm, 1997.

[10] A. K. Jonscher, "Dielectric Relaxation in Solids", Chelsea Dielectrics Press, London, 1983.

[11] N. Taylor, "Dielectric Response and Partial Discharge Measurement on Stator Insulation at Varied Low Frequency", PhD thesis, KTH, Stockholm, 2010. 